\title{
Linx
}

Revue des linguistes de l'université Paris X Nanterre

$41 \mid 1999$

L'hypothétique

\section{Une approche cognitive des conditionnelles : hypothèse et monotonie}

Francis Corblin

\section{OpenEdition}

\section{Journals}

Édition électronique

URL : http://journals.openedition.org/linx/757

DOI : $10.4000 / \operatorname{linx} .757$

ISSN : 2118-9692

\section{Éditeur}

Presses universitaires de Paris Nanterre

\section{Édition imprimée}

Date de publication : 1 décembre 1999

Pagination : 21-37

ISBN : 0246-8743

ISSN : 0246-8743

\section{Référence électronique}

Francis Corblin, «Une approche cognitive des conditionnelles : hypothèse et monotonie », Linx [En ligne], 41 | 1999, mis en ligne le 27 août 2012, consulté le 19 avril 2019. URL : http:// journals.openedition.org/linx/757 ; DOI : 10.4000/linx.757

Ce document a été généré automatiquement le 19 avril 2019

Département de Sciences du langage, Université Paris Ouest 


\title{
Une approche cognitive des conditionnelles : hypothèse et monotonie
}

\author{
Francis Corblin
}

1 Conditionnelle (au féminin) désigne dans cet article une représentation sémantique, non une structure syntaxique ou une forme du verbe. Notre objectif est d'éclairer ce qui définit les conditionnelles comme représentations sémantiques et d'en distinguer les sous-types.

2 Afin de fixer les idées d'emblée, nous illustrons à l'aide de phrases de forme si $P Q$ les principaux sous-types de conditionnelles que nous distinguons ${ }^{1}$ :

Conditionnelle factuelle :

Exemple : Si on chauffe de l'eau, elle bout.

Conditionnelles hypothétiques:

- éventuelle :

Exemple : Si Jean est chez lui, il regarde le match.

- contrefactuelle :

Exemple : Si on l'avait attaqué, il se serait défendu.

Conformément à la terminologie reçue en logique, dans si $\mathrm{P} \mathrm{Q}$, on appellera $\mathrm{P}$

l'antécédent de la conditionnelle, et $\mathrm{Q}$ le conséquent.

\section{L'analyse quantificationnelle des conditionnelles factuelles : Lewis (1975)}

\subsection{Sémantique}

3 L'article de Lewis (1975) introduit une approche quantificationnelle de certaines structures syntaxiques de forme si $P Q$. Considérons, pour illustrer, les deux phrases suivantes : 
(1) Si on chauffe de l'eau, en général, elle bout.

(2) Si on regarde la mer, on aperçoit parfois une voile.

Pour Lewis, la clé de la sémantique de ces phrases est l'adverbe de quantification représenté respectivement par en général et par parfois. Ces adverbes de quantification assertent qu'une certaine relation vaut entre les cas représentés par la subordonnée $(P)$ et les cas représentés par la principale $(Q)$. En substance, (1) asserte que la quasi totalité des cas dans lesquels on chauffe de l'eau est vrai sont aussi des cas dans lesquels l'eau chauffée bout ; (2) asserte que certainsdes cas dans lesquels on regarde la mer sont aussi des cas où l'on y aperçoit une voile.

5 Cette analyse permet d'intégrer ces conditionnelles dans le schéma général de la quantification, vu comme l'expression d'une relation entre un restricteur et un scope ${ }^{2}$; les adverbes de quantification expriment une relation entre un restricteur (le contenu de la subordonnée introduite par si) et un scope (le contenu de la principale). Dans cette manière de voir, la signification de la structure syntaxique si $P Q$ est entièrement subordonnée à un adverbe de quantification: $P$ délimite le restricteur de l'adverbe de quantification, et $Q$ son scope. S'il n'y a pas d'adverbe de quantification explicite, la quantification est interprétée selon Lewis comme une quantification (quasi) universelle.

Cette analyse s'applique correctement aux phrases en si dans lesquelles si est commutable avec quand :

(3) Quand on chauffe de l'eau, en général, elle bout.

(4) Quand on regarde la mer, on aperçoit parfois une voile.

Dans une conception classique de la sémantique, ces phrases ne soulèvent pas de problème particulier: elles sont vraies ou fausses selon que la relation exprimée par l'adverbe de quantification est vérifiée ou non. Si on a une connaissance totale du Modèle dans lequel on veut interpréter une phrase comme (1), on peut vérifier s'il est exact que la quasi-totalité des cas où on chauffe de l'eau sont des cas où cette eau bout. On appelle parfois ces conditionnelles « law-like» dans la mesure où elles sont aptes à exprimer des relations entre des types de faits dans un monde de référence. Nous les appellerons ici factuelles.

L'interprétation quantificationnelle des factuelles est résumée dans le schéma suivant :

\begin{tabular}{|l|l|}
\hline & Si P parfois Q \\
\hline & Si P en général Q \\
\hline Quantificateur & $\rightarrow$ Adverbe de quantification : parfois, en général... \\
\hline Restricteur & $\rightarrow \mathrm{P}$ \\
\hline \hline Scope & $\rightarrow \mathrm{Q}$ \\
\hline
\end{tabular}




9 Le propre des factuelles, par rapport à d'autres structures quantificationnelles (quantification sur les individus, par exemple) est qu'elles quantifient sur des procès, des éventualités et sur des instants, d'où l'utilisation de quand ou à chaque fois que. Pour une étude très approfondie de la sémantique des adverbes de quantification, on se reportera à De Swart (1993).

10 Cette analyse quantificationnelle présente quelques avantages sur l'analyse «propositionnelle» des factuelles, c'est-à-dire la traduction de si P Q par l'implication matérielle $\mathrm{P} \rightarrow Æ \mathrm{Q}$. Les factuelles ne sont plus liées à la sémantique de l'implication (stricte), ce qui permet de prendre en compte toute la gamme des adverbes de quantification. En outre, les adverbes de quantification relèvent de la quantification «non sélective" (Lewis), ce qui leur donne la possibilité de lier un nombre arbitraire de variables et de résoudre des difficultés connues et notamment le problème dit des « donkey sentences » (cf. Kamp, 1981, et Corblin, à paraître). Il est possible également de relier les structures sémantiques des factuelles à la quantification sur les individus en traitant les adverbes de quantification comme des quantificateurs représentables dans le cadre de la théorie des quantificateurs généralisés (Barwise et Cooper, 1981) en établissant des parallèles tels que :

\begin{tabular}{|l|l|}
\hline tout & toujours \\
\hline quelques & parfois \\
\hline aucun & jamais \\
\hline
\end{tabular}

(5) Tout homme travaille pour vivre.

Un homme travaille toujours pour vivre.

(6) Quelques hommes travaillent pour vivre.

Un homme travaille parfois pour vivre.

(7) Aucun homme ne travaille pour vivre.

Un homme ne travaille jamais pour vivre.

11 Cette analyse quantificationnelle conserve également quelques difficultés associées à la traduction des factuelles par une implication, et notamment le problème de savoir ce qu'il en est si le restricteur n'est pas vérifié: la prédiction des traductions logiques (propositionnelle ou quantificationnelle) est que la factuelle est vraie, alors que l'intuition linguistique, on le sait, n'est pas aussi catégorique.

\subsection{Cognition}

Si on adopte une perspective de modélisation des agents cognitifs, comment caractériser le statut des factuelles?

Un agent cognitif inclut une base de connaissances, laquelle contient au minimum une série de propositions particulières dont l'agent admet la vérité, c'est-à-dire une série de faits : $p, q, r, \ldots$

Les factuelles définissent des relations entre des types de faits, des régularités, au sens large, des règles; par exemple une factuelle si $P$ toujours $Q$ asserte qu'un fait de type $P$ ne peut pas être vérifié sans qu'un fait de type $Q$ correspondant ne soit également vérifié. 
Nous n'avons pas ici à nous prononcer sur la manière dont les factuelles « entrent » dans une base de connaissances, nous pouvons seulement admettre qu'en principe, il ne peut y avoir contradiction entre les factuelles et les faits inclus dans une base. Si par exemple un agent admet la factuelle si $P$ toujours $Q$, relation entre des faits de type $P$ et des faits de type $Q$, il ne peut pas entrer dans sa base de connaissances que $p_{i}$ est vrai et que $q_{i}$ est faux, $p_{i}$ représentant un fait de type $P$, et $q_{i}$ un fait de type $Q$. Si l'agent veut entrer $p_{i}$ et $q_{i}$, il lui faut changer la factuelle, qui deviendra, par exemple si $P$ presque toujours $Q$. On peut à cet égard voir les factuelles comme reflet de la structure de la base de faits : les factuelles notent les relations valides entre les types de faits représentés dans la base.

Considérons l'évolution d'une base de connaissances. Un agent cognitif est susceptible d'entrer dans sa base de nouveaux faits; ceux-ci lui sont proposés par ses capteurs, ou transmis par d'autres agents cognitifs. Plusieurs situations peuvent être distinguées.

Le fait proposé peut s'ajouter à la base de faits préexistante (celle-ci ne contient pas la négation de ce fait particulier), et l'adjonction de ce fait n'exige la modification d'aucune des règles (factuelles) existantes. On peut alors parler d'évolution monotone de la base de connaissances.

Les factuelles, d'autre part, sont des prémisses d'inférences qui permettront de déduire de nouveaux faits : si un agent admet une factuelle si $P Q$, et qu'il intègre $p_{i}$ à sa base de connaissances, il est en principe contraint d'admettre aussi $q_{i}$, faute de quoi il doit renoncer à la factuelle si $P Q$. Supposons, par exemple, qu'un agent cognitif admette la factuelle Si une abeille butine, elle fait du miel. Cet agent peut être amené à constater qu'une abeille $a$ butine. Si sa base de connaissances est rationnelle, c'est-à-dire si les faits et les règles ne sont pas contradictoires, sa base de connaissances doit aussi intégrer le fait que l'abeille $a$ fait du miel, faute de quoi, il devrait renoncer à la factuelle Si une abeille butine, elle fait du miel. Or un agent cognitif tend à considérer, par défaut, que l'acquisition d'un nouveau fait ne change pas les règles.

Essayons une approche un peu plus réaliste d'un agent cognitif. Il prend connaissance de son environnement par ses capteurs et admet la vérité d'un certain nombre de faits. Il abstrait de ces faits des règles qui mettent en relation ces faits. En somme, l'agent est dans la position suivante: "pour ce que je sais jusqu'à présent, les généralités $R$ sont valides ». Par exemple, «Jusqu'à présent, les abeilles qui butinent dont j'ai connaissance font du miel ». Si l'agent rencontre une nouvelle abeille butineuse, en théorie il pourrait s'agir d'une exception à cette règle, et en théorie donc, toutes les règles qui commencent par «A chaque fois qu'une abeille butine » devraient être suspendues, et l'agent devrait vérifier que l'abeille $a$ bien les propriétés mentionnées par ses règles avant de les maintenir en toute certitude. Or ce n'est pas ce qu'on observe chez un agent cognitif qui ressemblerait à un agent humain : dans ce cas, l'agent fonctionne en opérant ce qu'on pourrait appeler une présomption de monotonie. Cela signifie que l'agent admet par défaut qu'un nouveau fait ne modifie pas les règles. La nouvelle butineuse est donc intégrée sans modification de la règle ; bien que le conséquent l'abeille a fait du miel n'ait pas été observé empiriquement, il devient (au moins virtuellement) membre de la base de connaissances. 


\section{Des factuelles aux hypothétiques}

\subsection{Sémantique}

De nombreuses structures si $P Q$ du français n'admettent pas la sémantique précédemment mise en place pour les factuelles. Nous les avons isolées sous le nom d'hypothétiques, pour indiquer que c'est seulement eu égard à la base de connaissances d'un sujet que nous pouvons les rassembler. C'est même très précisément eu égard à la base de faits que nous pouvons les repérer et les distinguer.

Hypothétique éventuelle :

(8) Si Jean est chez lui, il regarde la télévision.

21 A la différence des factuelles, cette phrase ne porte pas sur des types de faits, mais sur des faits particuliers, à savoir que Jean soit chez lui au moment où est tenu le discours (8), et qu'il regarde la télévision à ce même instant. On peut repérer l'hypothétique éventuelle par le fait que ni l'antécédent de la conditionnelle $p$ ni sa négation $p$, n'appartiennent à la base de faits du locuteur. Autrement dit, on a affaire à une hypothétique éventuelle si $p q$ si le locuteur ne sait pas si $p$ ou si $p$. C'est dire que nous ne pouvons définir la catégorie des hypothétiques éventuelles que si nous adoptons une perspective épistémique, dans laquelle les locuteurs ont une connaissance partielle de l'univers de référence, et ignorent ce qu'il en est de la vérité de certains faits. A ce titre on sort du schéma classique dans lequel tout fait envisageable est nécessairement traité par le système comme vrai ou faux. Il faut ici admettre en outre des faits dont la valeur de vérité n'est pas connue.

Hypothétique contrefactuelle :

(9) Si on l'avait attaqué, il se serait défendu.

On repère en revanche les contrefactuelles par le fait que l'antécédent $p$ de si $p q$ doit nécessairement être considéré comme faux par le locuteur.

Le schéma valide jusqu'à présent pour les factuelles ne peut en aucun cas s'appliquer à ces phrases, dont la sémantique est, de plus, notoirement problématique. Il suffit, pour s'en convaincre, d'observer qu'il n'est en rien indiscutable que les hypothétiques puissent être dites vraies ou fausses. Le point n'est pas seulement qu'il serait difficile de dire si elles sont vraies ou fausses (ce qu'on dit souvent des contrefactuelles), il serait plutôt qu'en elle-même une hypothétique semble résister à l'attribution d'une valeur de vérité.

Partant d'une hypothétique éventuelle comme si $p q$, il est possible, par exemple d'apprendre qu'on a effectivement $p$ et $q$. Mais en déduira-t-on que l'hypothétique est (était?) vraie? Il semble qu'on soit prêt à utiliser de nombreux attributs (confirmé, juste, etc.), mais plus difficilement le mot vrai. D'autre part, si cette phrase était une proposition vraie ou fausse, on ne voit pas comment on pourrait l'analyser comme une fonction de vérité : si on applique le schéma des factuelles aux contrefactuelles, elles devraient être toujours vraies, et les hypothétiques recevront difficilement une valeur de vérité, car leur antécédent n'est ni vrai ni faux.

Pourtant, on observe que les hypothétiques utilisent dans de nombreuses langues les mêmes marqueurs formels (en l'occurrence, si $p q$ est en français une expression possible pour chacune d'elles). Il est donc naturel de chercher un lien entre elles. 

contient sont « ordonnés » en vertu de leur similarité vis-à-vis d'un « idéal » défini par la base elle-même. Prenons pour exemple une base épistémique, c'est-à-dire l'ensemble des faits reconnus pour vrais; pour Kratzer, ces mondes possibles sont ordonnés (ordre partiel) en fonction de leur conformité à l'« ordre normal des choses ».

31 L'idée centrale serait que les hypothétiques quantifient, non comme les factuelles sur des « cas », mais sur des mondes possibles. En substance, une éventuelle affirmerait que tous les mondes possibles (qui sont les plus proches de l'ordre normal des choses) dans lesquels $p$ est vrai sont des mondes dans lesquels $q$ est vrai. Cette approche est issue du point de vue de la logique modale, et elle semble au moins en mesure de réintégrer les hypothétiques dans une théorie sémantique connue. En principe donc, les hypothétiques seraient bien des propositions: elles seraient par conséquent vraies ou fausses. Les difficultés qu'elles posent viendraient éventuellement de la difficulté qu'il y a à évaluer des propositions qui quantifient sur des mondes possibles parce que nous ne savons pas très bien construire « tous les mondes possibles (conformes à l'ordre normal des choses) dans lesquels $p$ est vrai ", et encore moins les mondes possibles qui diffèrent du nôtre en ce qu'une proposition fausse dans notre monde est, dans ces mondes possibles, vraie.

Notre objectif n'est pas ici de critiquer cette théorie, mais nous allons essayer de proposer une approche cognitive des hypothétiques qui essaie de mieux rendre compte des intuitions qui accompagnent leur emploi en utilisant le point de vue esquissé plus haut. 


\subsection{Approche cognitive} nouveaux exprimés par l'énoncé du problème. Il est facile de voir comment la factuelle (11) peut justifier des hypothétiques dans ce cadre. De cette factuelle, on peut déduire des hypothétiques de forme :

(12) Si ce triangle A est équilatéral, chacun de ses angles mesure $60^{\circ}$. mises à jour de bases de faits : toute mise à jour d'une base intégrant le triangle A est équilatéral, est nécessairement une mise à jour qui intègre chacun des angles $d u$ triangle $A$ mesure $60^{\circ}$. 
Cette déduction d'hypothétiques à partir des factuelles s'obtient formellement comme une instanciation : une hypothétique si $p_{i} q_{i}$ instancie une factuelle de type si $P Q$ si si $p_{i} q_{i}$ instancie des variables de si P Q.

Pourquoi pouvons-nous être aussi affirmatif dans ce cas et dire que la factuelle « justifie » l'hypothétique qui l'instancie? C'est que nous sommes dans une base de connaissances mathématiques, base de connaissances dont l'évolution obéit à quelques contraintes dynamiques du type suivant: il existe un sous-ensemble de règles (universelles) intangibles, et la rationalité de la base (correspondance faits / règles) doit être parfaite. L'hypothèse est parfaitement "justifiée » parce qu'elle instancie une règle, et que cette règle strictement universelle n'est pas susceptible d'être révisée (affaiblie). Il est donc nécessaire que pour ce type de discours et de règles, toute révision intégrant $\mathrm{p}_{\mathrm{i}}$ soit une révision intégrant $\mathrm{q}_{\mathrm{i}}$. $\mathrm{S}$ 'il en était autrement, cela signifierait qu'on ne peut pas préserver la présence dans la base de la factuelle universelle si $P Q$ et de $p_{i}$ en sauvegardant la rationalité de la base.

Nous pouvons donc être certain dans ce cadre (dans ce type de discours) que la factuelle engendre une série d'hypothétiques valides.

Cette conception de l'hypothèse la met en relation étroite avec les schémas d'inférence et plus précisément le modus ponens. Si nous admettons la validité d'une factuelle si $\mathrm{P} Q$, alors, à chaque fois que nous mettrons à jour une base avec $\mathrm{p}_{\mathrm{i}}$ (instanciant $\mathrm{P}$ ), nous devons la mettre à jour avec $\mathrm{q}_{\mathrm{i}}$. Mais il est bien clair que ce schéma d'inférences lui-même n'est valide que dans un type de discours déductif, comme les mathématiques, type de discours dont les propriétés distinctives sont en l'occurrence les suivantes : les règles sont universelles, et intégrer un nouveau fait dans la base ne peut jamais conduire à réviser un certain ensemble de règles. En substance, si nous avons une série de factuelles dont l'antécédent est A est un triangle équilatéral, ces factuelles sont universelles, et si nous intégrons à l'occasion d'un problème un fait de type A est un triangle équilatéral, nous ne nous attendons pas à pouvoir modifier la série de règles préexistantes. En conséquence, toutes les règles universelles intangibles s'instancient en hypothétiques valides.

Pour bien comprendre les différences entre ce type de discours et le discours ordinaire, il suffit d'observer que le discours ordinaire repose sur des bases de connaissances où les factuelles utilisent toute la gamme des adverbes de quantification (en général, souvent, parfois, ...), et dans lesquelles les factuelles sont en général obtenues par induction à partir de faits observés. Ainsi, on peut avoir une factuelle de type quand Jean sort sous la pluie, il prend toujours son parapluie. Mais si on a une nouvelle occurrence de Jean sort sous la pluie, il est possible qu'il s'agisse d'un cas qui va nous inciter à affaiblir la règle (quand Jean sort sous la pluie, il prend presque toujours son parapluie).

On ne peut donc pas soutenir généralement que la présence d'une factuelle dans une base de connaissances valide une hypothèse qui l'instancie, si on réserve la notion de validation à un type de discours dont le modèle est fourni par les mathématiques.

En revanche, il peut être utile de généraliser la notion de justification :

(13) Justification :

Une hypothétique $\mathrm{H}$ est justifiée par rapport à une base de connaissances $\mathrm{B}$ si $\mathrm{H}$ est une instanciation d'un $\mathrm{F}$ appartenant à $\mathrm{B}$.

L'idée est ici que l'on distingue les cas où une base de données contient une factuelle instanciée par une hypothèse. Pour simplifier, on s'en tiendra uniquement à des factuelles universelles, mais il est clair que la notion de justification pourrait être 
modulée en fonction de l'adverbe de quantification des factuelles instanciées par l'hypothétique: selon que l'on a dans une base de connaissances s'il pleut, Jean prend toujours (souvent, parfois) son parapluie, l'hypothèse correspondante si Jean est sorti, il a pris son parapluie sera plus ou moins justifiée. Si, en revanche, nous avions la factuelle s'il pleut, Jean ne prend jamais son parapluie, l'hypothétique qui l'instancie ne serait pas justifiée. Mais pourquoi, au fond, distinguer les hypothétiques justifiées des autres? Très précisément sur la base suivante : les hypothétiques, de notre point de vue, expriment des relations entre des bases de connaissances; elles affirment que toute révision d'une base de connaissances intégrant $p_{i}$ intégrera $q_{i}$. Si l'hypothèse est justifiée, cela signifie que l'intégration de ces deux faits dans une base de connaissances est préservatrice des règles de cette base. Comme d'autre part, la base est cohérente avant mise à jour, nous savons que $q$ ${ }_{i}$ 'était pas dans cette base, et par conséquent que l'insertion de $q_{i}$ ''exigera pas le retrait de faits de la base. Si une hypothétique est justifiée, elle prédit donc simplement que l'acquisition éventuelle de $q_{i}$ représentera une mise à jour monotone de la base : aucune règle ni aucun fait précédemment admis n'auront à être retirés ou altérés.

Nous avons donc un parallélisme évident entre l'acquisition d'un fait et une hypothèse : l'acquisition d'un fait, nous l'avons dit plus haut, se fait sous "présomption de monotonie ». Cela signifie que lorsqu'un nouveau fait est admis dans une base, l'agent cognitif admet, par défaut, que les règles dont ce fait instancie une partie n'ont pas à être suspendues.

L'hypothèse éventuelle justifiée considère, elle, l'acquisition éventuelle d'un fait, et affirme que l'acquisition de ce fait, pour représenter une transition monotone, s'accompagnera de l'acquisition d'un autre fait.

Peut-être avons-nous là la source de l'idée d'ordre partiel des bases modales utilisée par Kratzer. Qu'est-ce-que l'ordre normal des choses pour une base de connaissances ? L'ordre normal des choses pourrait être que son évolution soit monotone, que des règles et faits admis n'aient pas à être retirés. Pour une base de connaissances déductives (de type mathématique), c'est simplement la seule évolution possible. Pour une base de connaissances empiriques, ce n'est pas tout à fait vrai : les faits admis sont reliés par des règles générales, obtenues souvent par induction (on n'a pas rencontré d'exception), mais ces règles pourraient être infirmées par de nouveaux faits. L'idée d'ordre normal des choses peut être assimilée à l'ensemble des règles: si une hypothétique est justifiée par une des ces règles, cette hypothétique fait seulement la prédiction que «l'ordre des choses » (les règles générales antérieurement valides) n'auront pas à être modifiées par l'acquisition éventuelle du fait antécédent de la conditionnelle, et que le conséquent sera également à inclure.

Dans cette approche, nous traitons les hypothétiques éventuelles comme une prédiction sur les mises à jour possibles par des faits nouveaux d'une base de connaissances. Pour définir une hypothétique, il faut donc prendre en compte une base de connaissances. L'hypothétique asserte que toute mise à jour éventuelle par $p_{i}$ et $q_{i}$ est monotone. La relation de justification (relation d'une hypothétique à une factuelle qui l'instancie) est une preuve que cette mise à jour éventuelle est monotone.

55 La relation de justification est nécessaire pour essayer de comprendre les inférences du type suivant : 
(14) S'il pleut, Jean sort toujours.

donc

S'il a plu, Jean est sorti. l'exemple des phrases concernant Hitler. Il est possible d'imaginer une base de connaissances contenant une factuelle telle que : Si un tyran gagne une guerre de conquête, il impose la langue de son pays d'origine aux pays conquis. Cette base étant cohérente, cela signifie qu'elle ne contient aucun fait contraire, par exemple aucun tyran vainqueur qui n'aurait pas imposé sa langue. Si par hypothèse nous voulons intégrer Hitler vainqueur dans la base, a priori nous ne pouvons plus savoir si la règle vaut, puisqu'elle est chargée de couvrir « un nouveau cas » qui aurait très bien pu la falsifier, s'il s'était produit.

Donc par principe, une contrefactuelle ne peut pas être justifiée dans le même sens qu'une éventuelle. Considérons une éventuelle si $p_{i} q_{i}$ justifiée par une factuelle dans une base de connaissances. La notion de mise à jour préservant la base a un sens : nous pouvons affirmer que toute intégration de $p_{i}$ intégrant en outre $q_{i}$ est préservatrice des règles de la base. En revanche, si nous avons une contrefactuelle si $p_{i} q_{j}$ justifiée par une factuelle d'une base, nous ne pouvons pas affirmer que toute intégration de $p_{i}$ intégrant $q_{i}$ est préservatrice des règles de la base. Néanmoins, on peut dire que la relation de justification vaut des deux types d'hypothétiques pourvu qu'on définisse la justification sur la seule base de l'intanciation d'une factuelle. 

par pourquoi. Ce que donne le locuteur pour asseoir sa prédiction est ici un peu indirect, mais nous pouvons suivre approximativement le chemin inférentiel. Pour établir que sa prédiction est justifiée, le locuteur donne une prémisse d'où on infère quelque règle de type: Si on invite Jean, Marie ne vient pas. Autrement dit, le locuteur veut établir que sa prédiction est justifiée par une règle jusqu'à présent valide dans la base de connaissances. Naturellement, aucune prédiction de ce type ne peut être assurée. Il pourrait se faire que on invite Jean et Marie vient soient intégrés à la base de faits, mettant ainsi fin à la validité de la règle. Le rôle de l'hypothétique est simplement d'affirmer qu'une prédiction concernant le devenir d'une base de connaissances est justifiée par les règles existantes de la base.

Poussée à l'extrême, notre proposition consisterait à voir dans chaque hypothétique la réaffirmation, ou l'activation, d'une factuelle qu'elle instancie. Si nous ne pouvons pas donner de valeur de vérité à une hypothétique, nous cherchons du moins à vérifier s'il existe bien une règle qui en ferait une hypothétique justifiée. 

arbitraire du locuteur :

(18) S'il a été assassiné, je suis sûr que c'est Jean qui a fait le coup.

Il est difficile, à première vue, de restituer une factuelle correspondante, mais la notion d'instanciation étant assez lâche, on pourrait s'appuyer sur des factuelles comme :

(19) A chaque fois qu'une horreur est commise, c'est Jean qui la commet. (Jean est un triste sire.)

(20) A chaque fois qu'il a été la victime de quelqu'un, c'est Jean qui a fait le coup. (Jean le persécute toujours.)

Il y a en fait, quand on « part » d'une hypothétique, un choix possible, et deux versions un peu différentes de la théorie :

- version forte : une hypothétique est mise en relation avec une factuelle qu'elle instancie, et qui la justifie ;

- version faible : une hypothétique est mise en relation avec une base de connaissances qui la justifie.

Il semble que la seconde version soit en définitive la plus exacte et la plus générale.

Quand nous partons d'une factuelle pour générer une hypothétique, nous pouvons identifier avec précision ce qui «justifie » l'hypothétique : si la loi générale est vérifiée dans la base, une évolution de la base qui instancie ses deux termes (antécédent et conséquent de la factuelle) sera nécessairement monotone.

Quand nous partons d'une hypothétique, il n'est pas certain que l'on puisse identifier une factuelle qui justifierait la prédiction que l'évolution éventuelle considérée est monotone. Peut-être avons-nous plutôt une configuration de type : rapportée à notre base de faits et de règles, l'évolution considérée est monotone (elle peut être effectuée sans enlever (ou affaiblir) de règle, et sans modifier autrement les faits admis).

Il est ainsi possible qu'une justification se fonde sur l'invocation de faits et non de règles. Dans l'exemple (18), si les deux personnages sont sur une île déserte, il est clair que l'évolution envisagée est monotone. Si ce n'est pas Jean qui a fait le coup, et qu'il y ait eu néanmoins assassinat, il faudra enlever de la base le fait que les deux personnages sont sur une île déserte.

comprend alors mieux comment et sur quelle base les locuteurs peuvent discuter les hypothétiques. Il s'agit, à chaque fois de donner des arguments pour établir, ou infirmer, que la transition est monotone, et ce discours consistera à repasser sur les termes de la base de connaissances et à mentionner ou les règles qui justifient, ou des faits qu'il faudrait enlever pour intégrer la transition éventuelle, etc. En ce sens, une hypothétique peut être vue comme une activation d'éléments particuliers d'une base de connaissances, effectuée le plus souvent, à partir d'une question « qui se pose » et qui est exprimée par le conséquent de la conditionnelle.

Considérons l'exemple suivant :

A : Crois-tu que Pierre a regardé le J.T. ce soir?

$B: S$ 'il est rentré de voyage, je suis sûr qu'il a regardé le J.T.

Ici, le locuteur $B$ répond à la question posée de manière indirecte, en faisant d'une réponse positive le conséquent d'une éventuelle. C'est une manière de donner une 
condition (rentrer de voyage) qui, dans l'ordre normal des choses (donc en vertu d'une base de connaissances) s'accompagne de la vérité du fait (regarder le J.T.). Il se peut que l'incertitude quant au retour de Pierre soit plus faible et que par conséquent, dans l'ordre normal des choses, une réponse à la question soit moins incertaine. Dans cet exemple, la partie de la base de connaissances « activée » est relative aux habitudes de Pierre, et peut être approchée par Quand Pierre rentre de voyage, il regarde le J.T., ou Quand Pierre est chez lui, il regarde le J.T.

\section{Conclusion}

Cet article tente de caractériser les hypothétiques en tant que structures conditionnelles, et plus particulièrement d'expliciter le lien entre les conditionnelles factuelles et les conditionnelles hypothétiques, qui empruntent souvent, dans les langues naturelles, des moyens d'expression communs, notamment les structures si $P Q$.

Pour ce faire, nous avons été amené à essayer d'éclairer le statut des structures hypothétiques. Dans l'esprit de Ramsey et Stalnaker, nous avons admis qu'une hypothétique est une prédiction sur l'évolution d'une base de connaissances: la prédiction que toute évolution intégrant l'antécédent intégrera le conséquent. Nous avons en outre supposé qu'une hypothétique repose sur une présomption de monotonie : une hypothétique asserte que toute évolution qui intègre l'antécédent et le conséquent est monotone (n'affecte pas les règles de la base de connaissances). Dans cette approche cognitive, la relation fondamentale d'une hypothétique à une base de connaissances est une relation de justification : une factuelle justifie les hypothétiques qui l'instancient, et une hypothétique doit être justifiée par une base de connaissances. Une hypothétique est donc plutôt vue comme une activation d'une sous-partie des connaissances acquises (celles qui la justifient), que comme un pari sur les possibles.

\section{BIBLIOGRAPHIE}

BARWISE, J. et COOPER, R. (1981), « Generalized quantifiers and natural language », in Linguistics and Philosophy, 4, pp. 159-219.

BAUERLE, R., EGLI, U. et VON STECKOW, A. (1979), Semantics from Different Points of Views, Berlin, Springer.

CORBLIN, F. (à paraître), Approches formelles de la sémantique nominale. Représentation du discours et quantification généralisée.

DE SWART, H. (1993), Adverbs of Quantification. A Generalized Quantifier Approach, Garland Publishing.

FRANK, A. (1996), Context Dependence in Modal Constructions, Thèse, Université de Stuttgart.

GARDENFORS, P. (1988), Knowledge in Flux: Modeling the Dynamics of Epistemic States, Bradford Book, M.I.T. Press. 
KASHER, A. (1976), Language in Focus Foundations, Methods and Systems, Dordrecht, Reidel.

KEENAN, E. L. (1975), Formal Semantics of Natural Language, Cambridge University Press.

KRATZER, A. (1979), « Conditional necessity and possibility », in R. BAUERLE, pp. 117-147.

KRATZER, A. (1991), « Modality / Conditionals », in A. VON STECKOW et D. WUNDERLICH (eds), Semantik. Ein internationales Handbuch der zeitgenössischen Forschung, Berlin, pp. 639-656.

LEWIS, D. (1973), Conterfactuals, Oxford, Basil Blackwell.

LEWIS, D. (1975), « Adverbs of quantification », in KEENAN (1975).

MUNITZ, M. et UNGER, P. (1974), Semantics and Philosophy, New York University Press.

RAMSEY, F.P. (1931), The Foundations of Mathematics and Other Logical Essays, R.B. BRAITHWAITE, (ed.), Routledge et Kegan Paul.

RESCHER, N. (1964), Hypothetical Reasoning, Amsterdam, North Holland.

RESCHER, N. (1968), Studies in Logical Theory, Oxford, Basil Blackwell.

STALNAKER, R. (1968), « A Theory of conditionals », in RESCHER, pp. 98-112.

STALNAKER, R. (1974), « Pragmatic presupposition », in MUNITZ, pp. 197-213.

STALNAKER, R. (1976), « Indicative conditionals », in KASHER, pp. 179-196.

\section{NOTES}

1. Nous admettons évidemment que des phrases qui ne sont pas de structure si $P Q$ puissent être représentées comme des conditionnelles.

2. Je choisis ici de conserver de calques des termes anglais restrictor et scope afin de renvoyer sans ambiguïté à l'analyse de la quantification due à David Lewis. Tous les termes qui paraissent fournir une adaptation française (domaine, portée) sont en fait déjà utilisés dans tant d'autres acceptions qu'ils risqueraient ici de se comporter en faux amis.

\section{AUTEUR}

\section{FRANCIS CORBLIN}

URA 1028 CNRS

U.F.R. de Langue Française

Université de Paris IV

1 , rue Victor Cousin

75230 - PARIS Cedex 05 